\title{
CAPÍTULO 47: A ESCOLA COMO CAMPO DE ATUAÇÃO NO FORTALECIMENTO DA ALIMENTAÇÃO SAUDÁVEL: UMA REVISÃO INTEGRATIVA
}

\section{CHAPTER 47: SCHOOL AS A FIELD OF ACTIVITY IN STRENGTHENING HEALTHY FOOD: AN INTEGRATIVE REVIEW}

Fernanda Tayla de Sousa Silva ${ }^{1}$; Roseane Saraiva de Santiago Lima²; Maria de Fátima Costa Carneiro ${ }^{3}$; Ana Thaís Campos de Oliveira ${ }^{4}$ Ana Paula Ferreira de Almeida ${ }^{5}$.

\begin{abstract}
Resumo
Introdução: A formação de hábitos e práticas comportamentais acontece principalmente durante a infância, sendo importante o desenvolvimento de ações educativas, sobretudo de Educação Alimentar e Nutricional a fim de melhorar as condições de saúde. Objetivo: Identificar a produção científica que abordasse ações de promoção da alimentação saudável realizadas em escolas. Métodos: Trata-se de uma revisão integrativa da literatura. Os dados foram obtidos através da busca eletrônica nas bases de dados Literatura Latino-Americana e do Caribe em Ciências da Saúde (LILACS), PubMed e a Scientific Electronic Library Online (SciELO). Após a identificação dos artigos científicos, foram realizadas as seguintes etapas de seleção: a leitura criteriosa dos títulos, dos resumos e na íntegra. Resultados: A amostra consistiu em 13 artigos. Discussão: Os temas mais trabalhados foram alimentação saudável, consumo de frutas, legumes, leites e derivados, pirâmide alimentar, planejamento de cardápio saudável, higiene dos alimentos e utensílios, quantidade de óleo e sal das preparações e opções de lanches saudáveis. Conclusão: Os achados citam a escola como um espaço favorável para realizar ações de promoção da alimentação saudável, ressaltando a importância dos gestores e a comunidade escolar terem o compromisso de colocar em prática os programas de alimentação e nutrição.
\end{abstract}

Palavras-Chave: Educação alimentar e nutricional, Educação infantil, Promoção da saúde.

\begin{abstract}
Introduction: The formation of habits and behavioral practices happens mainly during childhood, being important the development of educational actions, especially of Food and Nutrition Education in order to improve health conditions. Objective: To identify scientific production that addressed actions to promote healthy eating in schools. Methods: This is an integrative literature review. The data were obtained through electronic search in the databases of Latin American and Caribbean Literature in Health Sciences (LILACS), PubMed and the Scientific Electronic Library Online (SciELO). After the identification of scientific articles, the following selection steps were carried out: careful reading of titles, abstracts and in full. Results: The sample consisted of 13

\footnotetext{
${ }^{1}$ Mestranda em Tecnologia de Alimento, Instituto Federal de Educação, Ciência e Tecnologia do Ceará (IFCE) tayliinhaf@gmail.com

${ }^{2}$ Mestre pelo Programa Saúde da criança e do adolescente, Universidade Estadual do Ceará (UECE) roseane.saraiva@ifce.edu.br

3 Bacharel em Nutrição, Instituto Federal de Educação, Ciência e Tecnologia do Ceará (IFCE) fati276@yahoo.com.br

${ }^{4}$ Mestranda em Tecnologia de Alimento, Instituto Federal de Educação, Ciência e Tecnologia do Ceará (IFCE) euthaiscampos.alimentos@gmail.com.br

${ }^{5}$ Mestranda em Tecnologia de Alimento, Instituto Federal de Educação, Ciência e Tecnologia do Ceará (IFCE) ana.paula.ferreira.almeida123@gmail.com.br
} 
articles. Discussion: The most discussed topics were healthy eating, consumption of fruits, vegetables, milk and dairy products, food pyramid, planning a healthy menu, hygiene of food and utensils, quantity of oil and salt in the preparations and options for healthy snacks. Conclusion: The findings cite the school as a favorable space to carry out actions to promote healthy eating, highlighting the importance of managers and the school community being committed to putting food and nutrition programs into practice.

Keywords: Food and nutrition education, Early childhood education, Health promotion.

\section{Introdução}

A Educação Alimentar e Nutricional (EAN) objetiva assegurar o Direito Humano à Alimentação Adequada (DHAA) e promover a Segurança Alimentar e Nutricional (SAN). A EAN conceitua-se como um campo de conhecimento de prática contínua e permanente, transdisciplinar, intersetorial e multiprofissional que visa a promoção da saúde através da adoção de hábitos alimentares saudáveis de forma autônoma e voluntária (BRASIL, 2012). A garantia do DHAA envolve, muito além da oferta de alimentos saudáveis, implica em respeitar os hábitos alimentares e viabilizar o acesso aos meios necessários para a aquisição do próprio alimento e da sua família, seja através do trabalho no campo ou na cidade (GREENWOOD; FONSECA, 2018). A EAN preocupa-se com o consumo consciente, questões ligadas a sustentabilidade, ao sistema de produção, abastecimento e distribuição, desse modo, incentiva o consumo de alimentos que geram pouco impacto ao meio ambiente, que valorize a cultura alimentar e priorize a produção local (TRICHES, 2015).

O conceito de DHAA refere ao direito humano de que todas as pessoas devem ter acesso regular, permanente, diretamente ou por meio de aquisições financeiras, a alimentos seguros e saudáveis, em quantidade e qualidade suficientes (BURITY et al., 2010). A Lei Orgânica de Segurança Alimentar e Nutricional LOSAN (Lei nº 11.346, de 15 de setembro de 2006) ressalta que SAN deve estimular práticas alimentares promotoras de saúde, respeitando a diversidade cultural, a economia e o meio ambiente, enfatiza que o acesso a alimentos de qualidade não deve comprometer o acesso a outras necessidades essenciais (BRASIL, 2006).

A alimentação equilibrada ao longo da vida é fundamental para garantir o crescimento e o bom funcionamento fisiológico, bem estar e manutenção da saúde. Tratando-se especialmente da infância, período marcado pela formação dos hábitos alimentares é imprescindível que pais e educadores estimulem os bons hábitos desde cedo já que é nessa fase que ocorre o desenvolvimento dos aspectos motor, cognitivo e afetivo 
da criança, em vista disso é um período que merece atenção e cuidados (ARAUJO et al., 2018; ALVES et al., 2020).

Sendo assim, uma boa alimentação deve tornar-se uma prática desde a infância e perpetuar-se ao longo de toda a vida. Para que crianças e os adolescentes adquiram um estilo de vida saudável na fase adulta, é fundamental que sejam orientados precocemente a adoção de bons hábitos (RECH et al., 2016). Conforme Alves et al. (2020) a alimentação é primordial para o desenvolvimento da criança, e é nesse período onde ocorre a formação de grande parte das potencialidades humanas, ou seja, qualquer distúrbio que ocorra nessa fase pode gerar sérias consequências na vida adulta.

De acordo com Araújo et al. (2017) nas últimas décadas, observa-se no Brasil um aumento significativo da prevalência de sobrepeso e obesidade em todas as faixas etárias e grupos sociais, modificações no estilo de vida, como alimentação inadequada, redução do gasto calórico diário são fatores que esclarecem o crescimento acerelado desse quadro no país. O excesso de peso é considerado fator de risco para Doenças Crônicas Não Transmissíveis (DCNT), assim tornando-se necessário a implementação de políticas públicas voltadas a prevenção e tratamento da obesidade, objetivando o esclarecimento da população quanto ao consumo de alimentos saudáveis, modificar padrões de comportamento alimentar e estimular a prática de atividade física.

O ambiente escolar é citado como um espaço promissor para a realização de atividades educativas, sobretudo de EAN para promoção de hábitos saudáveis e consequentemente a prevenção e controle do sobrepeso e obesidade. A educação possibilita a ampliação do conhecimento e desenvolvimento intelectual, tornando os indivíduos conscientes dos seus direitos e deveres sociais, além de críticos no momento de fazer escolhas alimentares. A inclusão da educação alimentar na escola torna-se fundamental visto que a ingestão excessiva e inadequada de nutrientes por falta de informações e acesso a alimentos de qualidade, viola o direito humano à alimentação saudável (GREENWOOD; FONSECA, 2018). Em 2007 foi instituído pelo Ministério da Saúde, através do Decreto n ${ }^{\circ}$ 6.286/2007, o Programa Saúde na Escola (PSE), no qual refere-se à promoção da saúde e prevenção de agravos à saúde, ressaltando a importância de práticas alimentares saudáveis no ambiente escolar (ARAÚJO et al., 2017; BRASIL, 2007).

Segundo Santos (2012) às intervenções de educação alimentar devem possuir abordagens e metodologias transformadoras que assumam uma perspectiva problematizadora, ultrapassando a visão somente instrumental e instrucional da educação. Ressaltando que as práticas educativas devem ser desenvolvidas de forma interdisciplinar 
a fim de desenvolver a autonomia dos indivíduos. Destaca-se o professor como papel chave nesse processo ensino-aprendizagem, e as ações em educação devem ultrapassar a educação formal de modo que o conjunto de práticas sejam capazes de transformar o ambiente no qual os indivíduos estão inseridos (TRICHES, 2015).

Portanto, as atividades de EAN desenvolvidas no ambiente escolar, devem envolver toda a comunidade escolar, não somente os alunos, por meio da implementação de estratégias educativas em conjunto com o projeto pedagógico das escolas. A alimentação escolar na rede públicas deve ser formulada segundo as diretrizes do Programa Nacional de Alimentação Escolar (PNAE), que além de assegurar assistência alimentar aos alunos com o emprego de alimentos saudáveis e adequados, incentiva a inclusão de ações de educação alimentar e nutricional no processo ensino-aprendizagem, dessa forma envolvendo toda a comunidade escolar (CAMOZZI et al. 2015; BRASIL, 2009).

Nesse contexto, através desse estudo objetivou-se identificar a produção científica que abordasse ações de promoção da alimentação saudável realizadas no ambiente escolar, com o propósito de responder a seguinte pergunta norteadora: o ambiente escolar é um espaço favorável para as ações da promoção da saúde e educação alimentar?

\section{Desenvolvimento}

\section{Metodologia}

Realizou-se uma revisão integrativa da literatura, que é um método de análise e síntese de informações de outros estudos de forma ampla e sistemática que busca identificar, analisar e discutir métodos e resultados de outras pesquisas possibilitando assim, gerar um conhecimento mais aprofundado sobre um tema investigado (SOUSA et al., 2017). Consiste em seis etapas distintas: elaboração da pergunta norteadora, amostragem, coleta de dados, análise crítica dos estudos incluídos, interpretação dos resultados e apresentação da revisão integrativa (DE SOUZA; DA SILVA; CARVALHO, 2010).

Desse modo foi realizado um levantamento bibliográfico com o intuito de analisar publicações sobre a promoção da alimentação saudável no ambiente escolar. $\mathrm{O}$ levantamento de dados foi feito através da busca eletrônica nas bases de dados Literatura Latino-Americana e do Caribe em Ciências da Saúde (LILACS), PubMed e a biblioteca eletrônica Scientific Eletronic Library Online (SciELO).

A busca de dados foi realizada pelos pesquisadores no período de maio a junho de 2018. Para a coleta dos estudos utilizou-se a combinação dos seguintes descritores 
"Educação Nutricional" AND "Escolares" e os termos em inglês "Nutrition Education" AND "School" AND "School Health Services" nas bases de dados citadas anteriormente.

Para a seleção dos estudos foi atribuído alguns critérios de elegibilidade: estudos que tratavam do tema em questão, publicações do período de 2013 a 2018 no idioma português, inglês e espanhol que tivessem disponíveis na íntegra online e fossem artigos gratuitos. Como critérios de exclusão estudos que não abordavam a temática proposta, estudos onde as intervenções não eram realizadas em escolas, revisão de literatura, publicações no formato de monografias, dissertações e teses.

Após a identificação dos artigos científicos, foram realizadas as seguintes etapas de seleção: a leitura criteriosa dos títulos e resumos, excluindo-se os estudos que não tratavam da questão de interesse. Os estudos duplicados nas bases de dados eram excluídos utilizando-se o programa Microsoft Excel versão 2016 que possibilitou a organização e sistematização dos trabalhos. As publicações consideradas elegíveis eram lidas na íntegra e avaliadas segundo o instrumento para coleta de dados validado por Ursi, 2005 que permite organizar as informações dos trabalhos em identificação, tipos de publicações, características metodológicas dos estudos e avaliação do rigor metodológico. Após essa análise os trabalhos eram incluídos nesta revisão (SOUSA et al., 2017).

Foram encontrados 247 artigos, mas somente 13 atenderam aos critérios de elegibilidade. Na figura 1 a seguir, mostra o fluxograma das etapas de seleção dos trabalhos incluídos no estudo. 


\section{Resultados}

Figura 1. Fluxograma das etapas de seleção

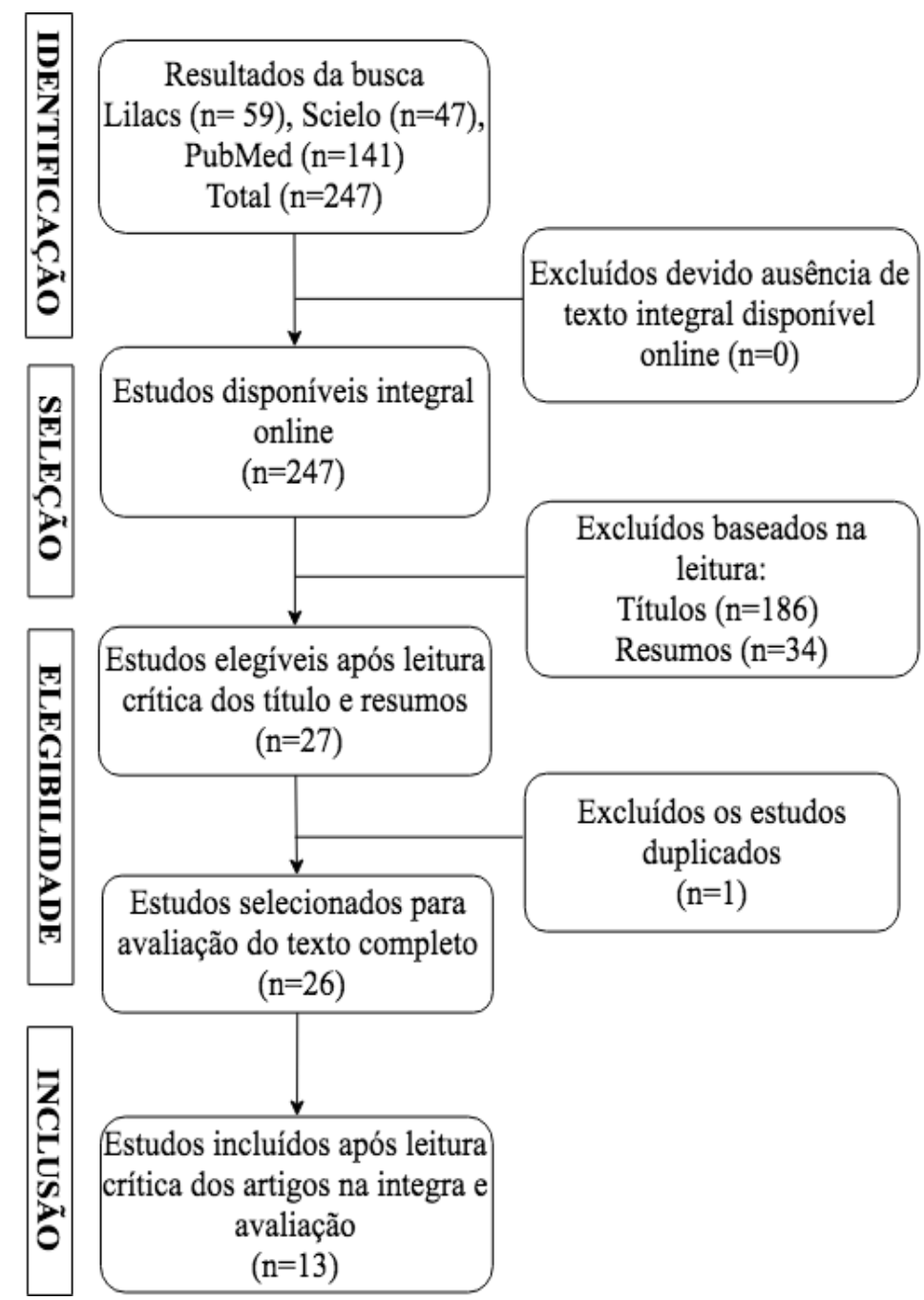

Fonte: Própria (2018).

Os artigos científicos foram respectivamente organizados e descritos no Quadro 1 a seguir conforme autor/ano, objetivos, população, intervenções e principais resultados. Dentre os trabalhos científicos selecionados, 7 apresentaram-se em língua inglesa, 4 na língua portuguesa e 2 na língua espanhola. 


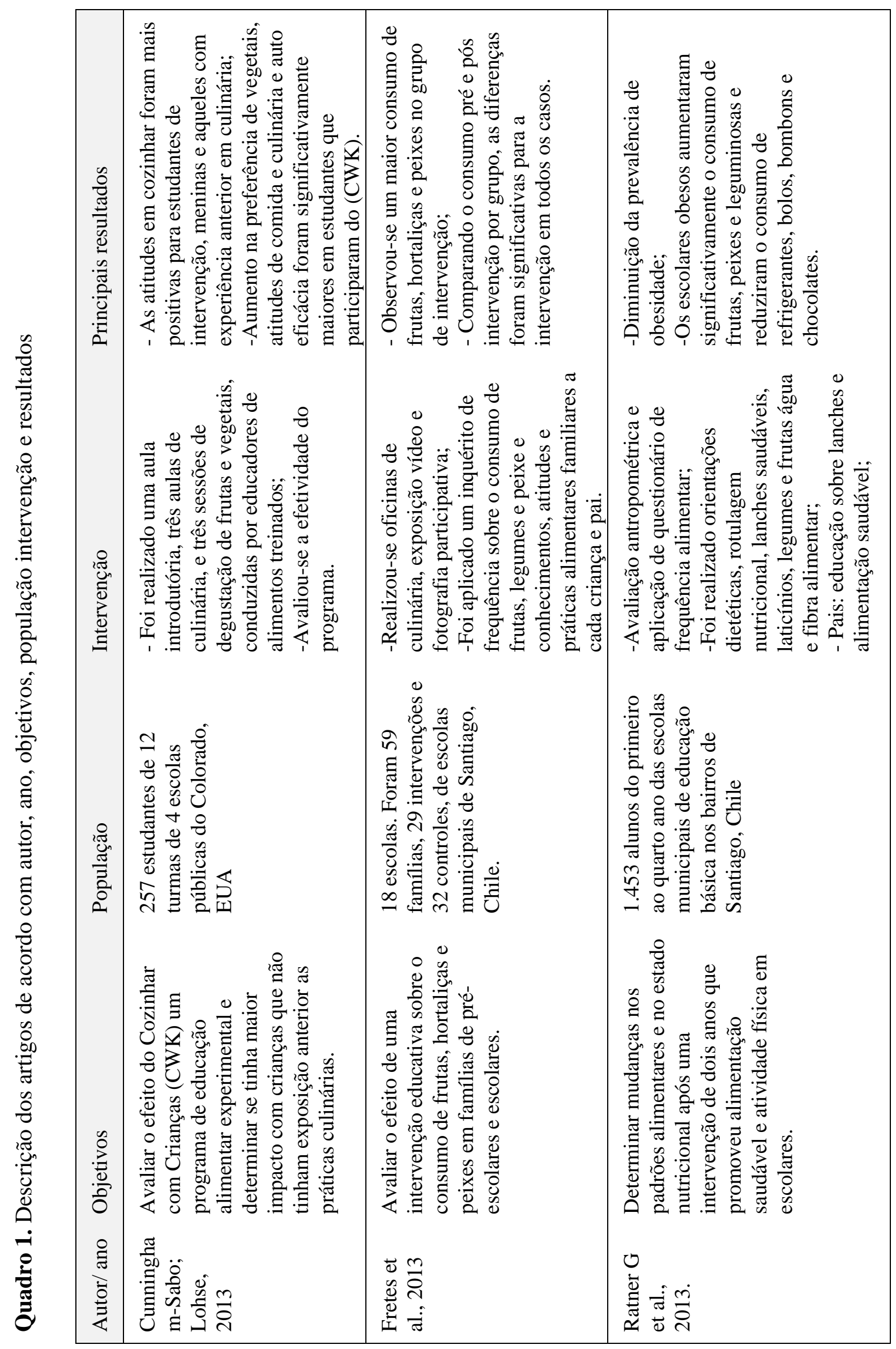




\begin{tabular}{|c|c|c|c|}
\hline 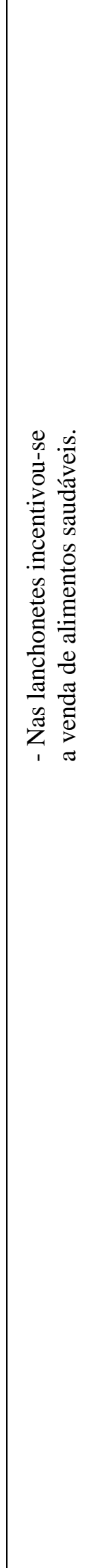 & 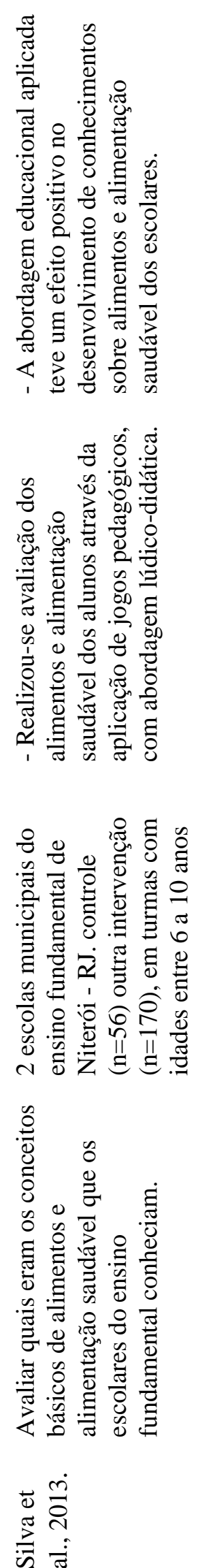 & 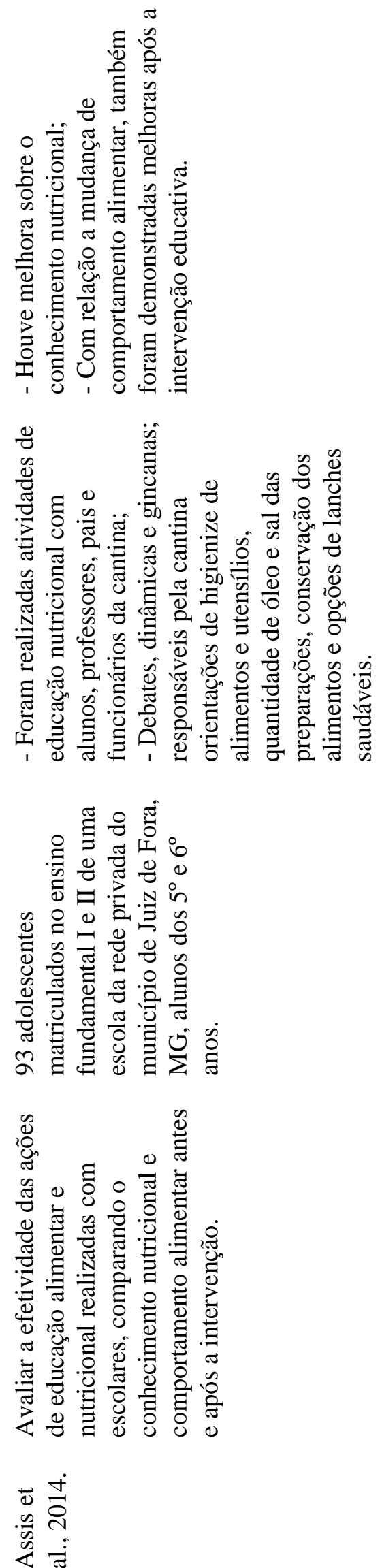 & 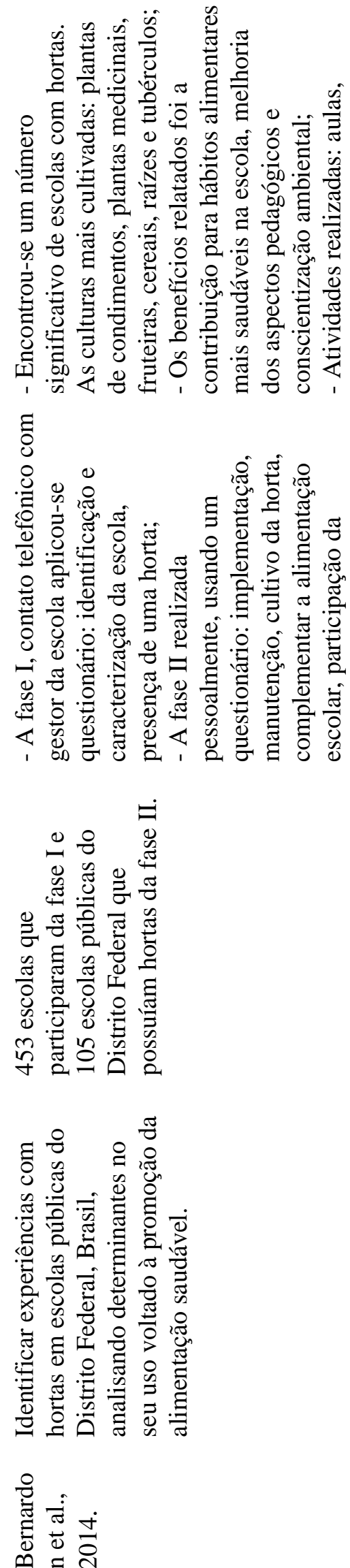 \\
\hline
\end{tabular}

[738] 


\begin{tabular}{|c|c|c|}
\hline 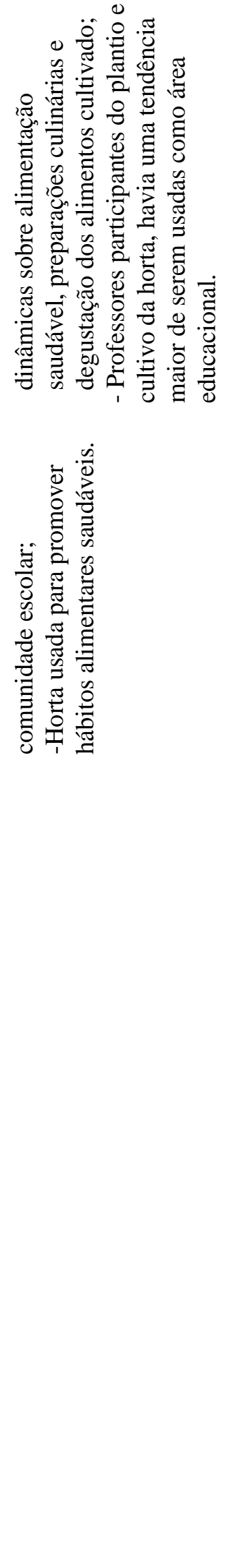 & 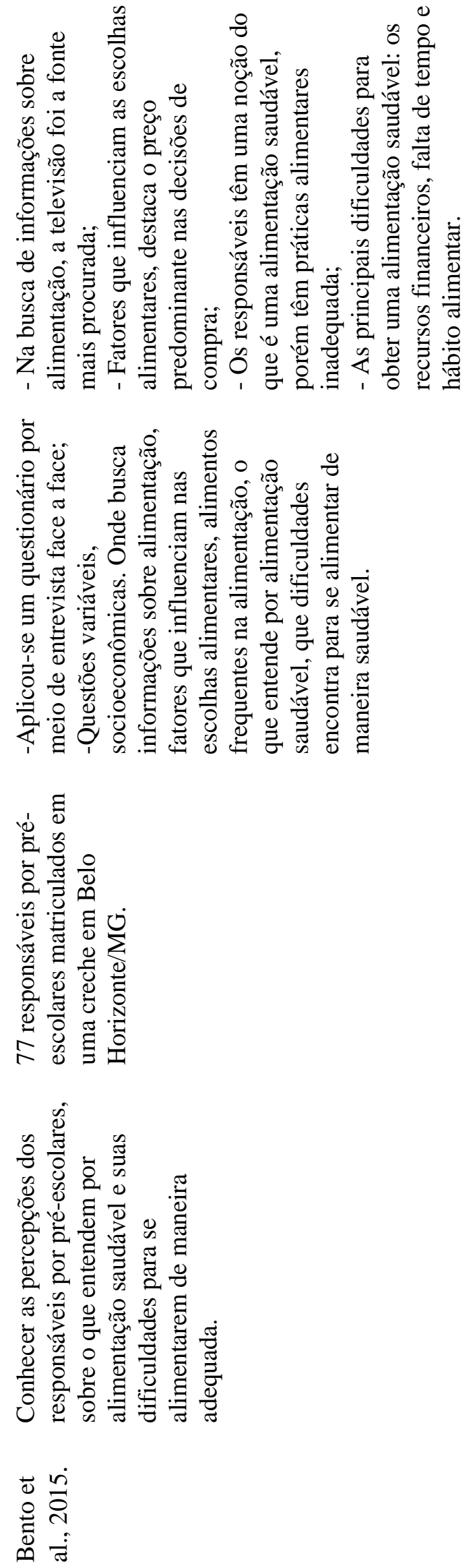 & 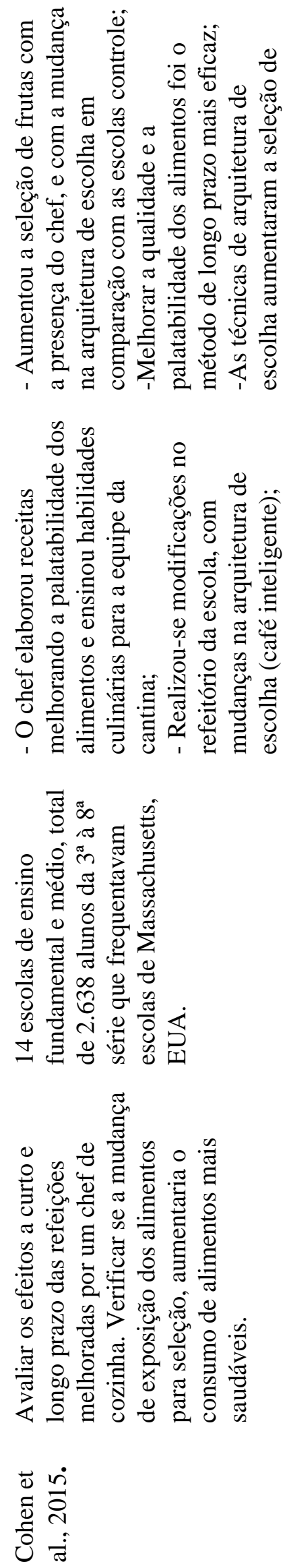 \\
\hline
\end{tabular}

[739] 


\begin{tabular}{|c|c|c|}
\hline 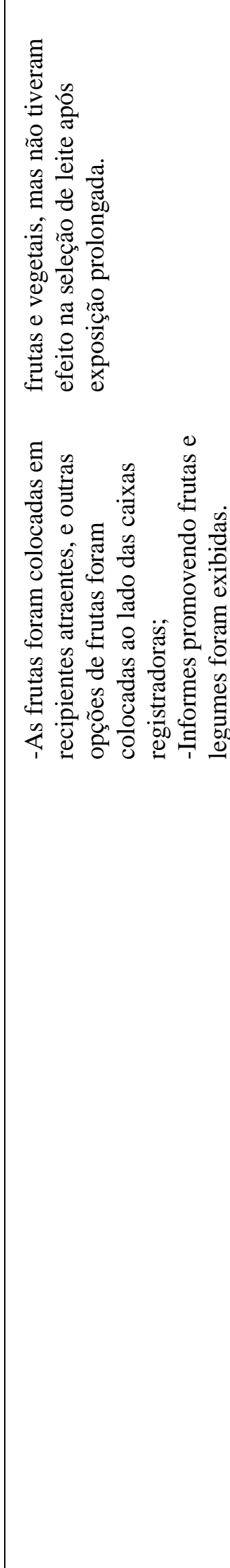 & 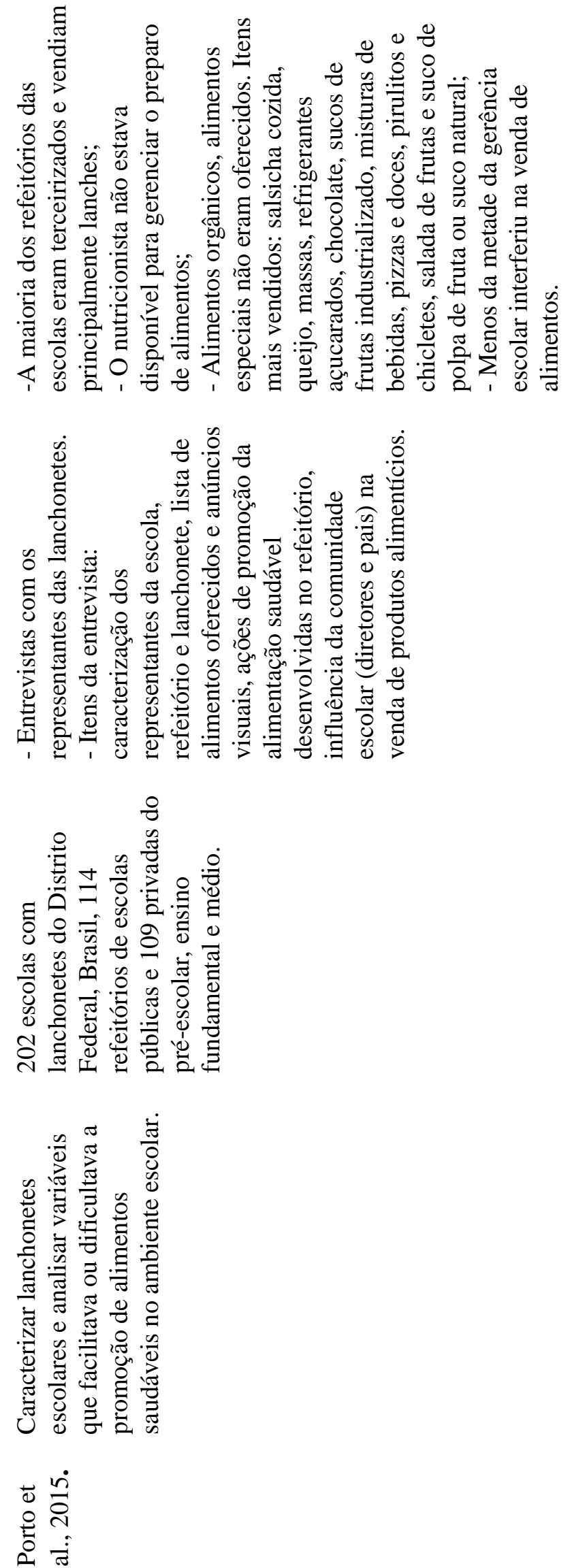 & 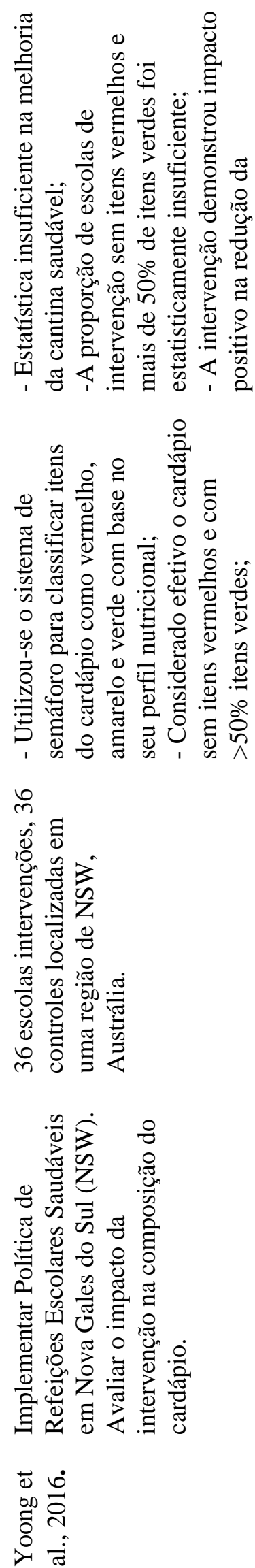 \\
\hline
\end{tabular}

[740] 


\begin{tabular}{|c|c|c|}
\hline 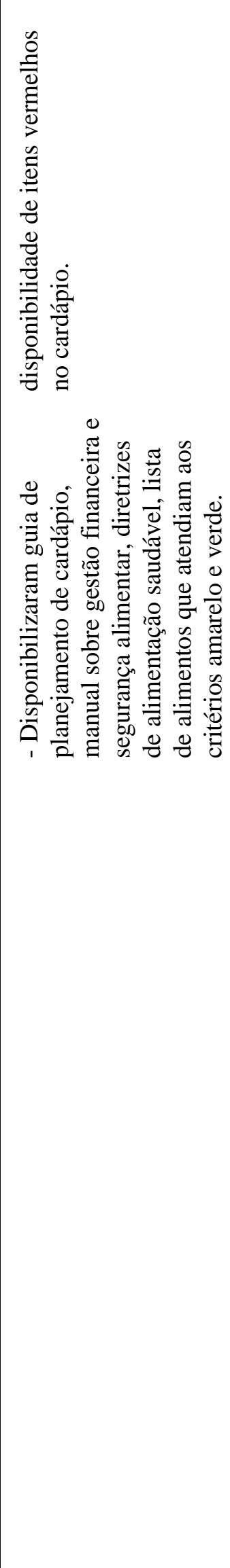 & 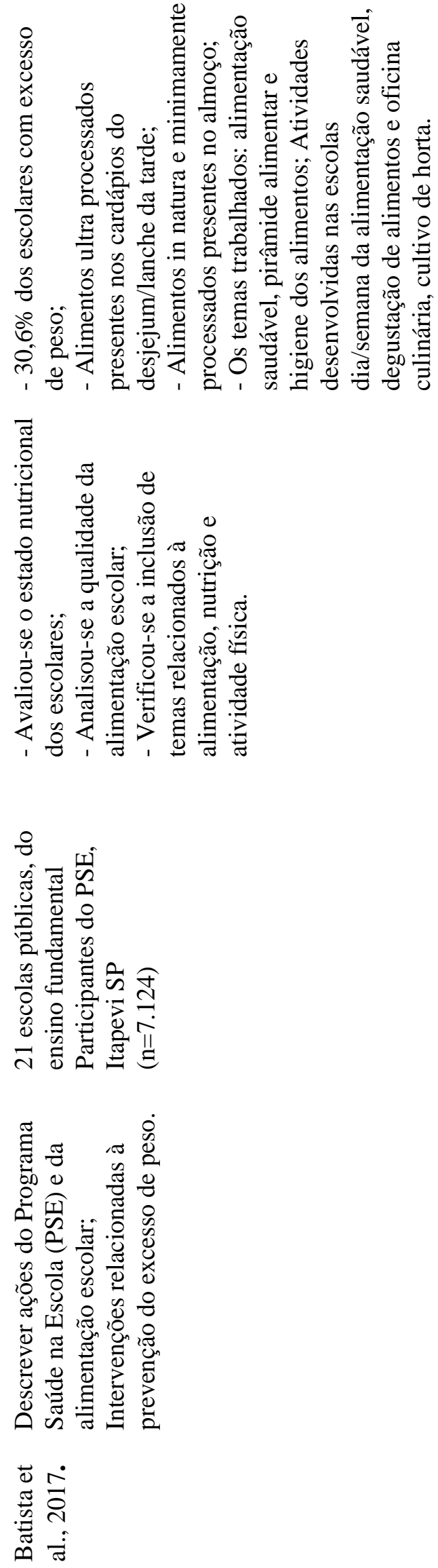 & 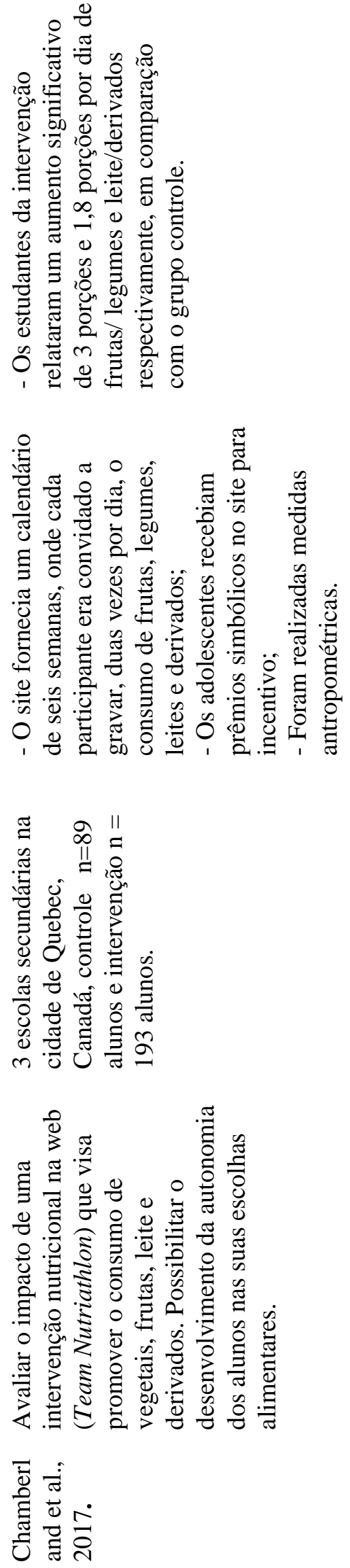 \\
\hline
\end{tabular}

[741] 


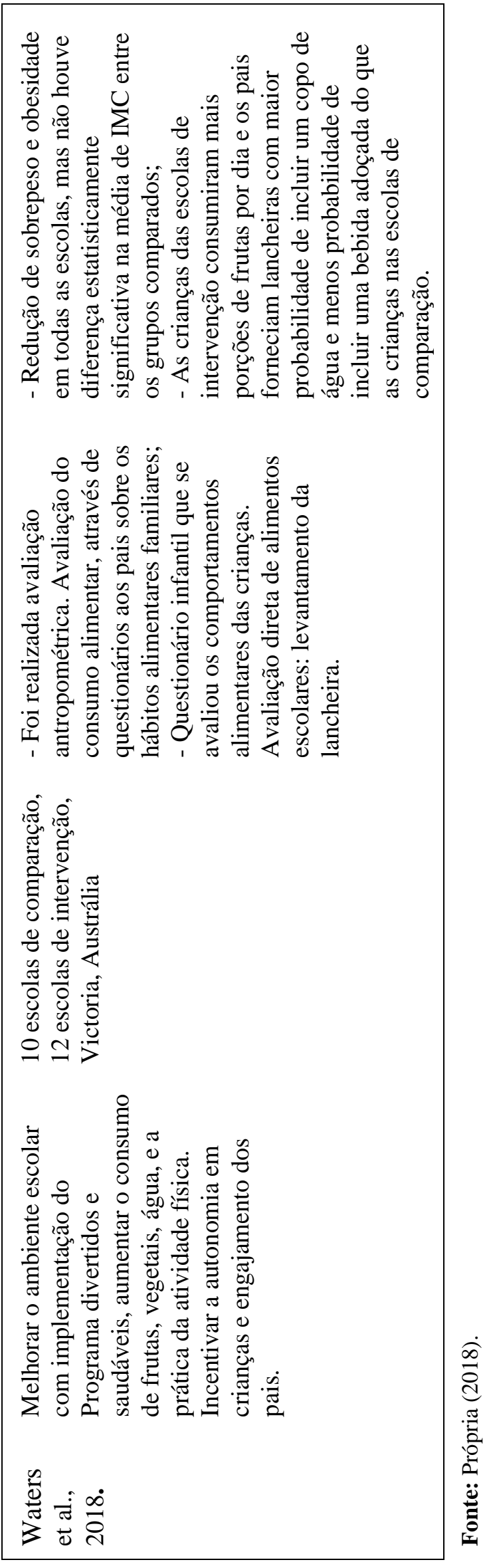

[742] 


\section{Discussão}

As ações de educação alimentar e nutricional realizadas pelos estudos citados anteriormente utilizaram as seguintes metodologias: aulas expositivas, palestras, debates, dinâmicas, gincanas, oficinas culinárias, degustação de frutas e vegetais, atividades lúdicas, exposição de vídeos, cartazes, mudanças na arquitetura de escolha dos alimentos, melhora da palatabilidade da merenda escolar, jogos lúdico-didático, jogos web e cultivo de hortas escolares. Os temas mais trabalhados foram alimentação saudável, consumo de frutas, legumes, leites e derivados, pirâmide alimentar, planejamento de cardápio saudável, higiene dos alimentos e utensílios, quantidade de óleo e sal das preparações e opções de lanches saudáveis.

Dentre as técnicas para verificação do sucesso das ações, ressalta-se avaliação antropométrica, peso, estatura e IMC, levantamento do consumo alimentar através da aplicação de inquéritos alimentares realizados antes e após as intervenções, cálculo de resto-ingesta, observações visuais e aplicação de questionários padronizados. Destaca-se o sistema de semáforo, jogos lúdicos didáticos e os jogos web, mudança na arquitetura de escolha dos alimentos as metodologias mais interativas e inovadoras entre os estudos.

As metodologias dos estudos citados anteriormente como cozinhar com crianças, orientações nutricionais com pais, crianças e donos das lanchonetes, cultivo de hortas escolares, melhorar a palatabilidade dos alimentos através de um chefe de cozinha e intervenção nutricional na web, são as abordagens que obtiveram os resultados mais significativos (CUNNINGHAM-SABO; LOHSE, 2013; RATNER et al., 2013; BERNARDON et al., 2014; COHEN et al., 2015; CHAMBERLAND et al., 2017). Referindo ao estudo Chamberland et al. (2017) apontam que os adolescentes são mais receptivos ao uso de ferramentas online, pois foram criados na evolução da tecnologia, mostrando-se uma boa metodologia para ser usada com esse público alvo.

Em contrapartida as metodologias que realizam somente antropometria, aplicação de questionários e atividades expositivas geram menos impacto quanto a mudança de hábitos alimentares e estilo de vida saudável pois, estimula menos a atenção do público alvo, dificultando assim, o processo de aprendizagem. No estudo Bento et al. (2015) realizaram aplicação de questionário semiestruturado a fim de conhecer a percepção dos pais dos escolares sobre alimentação saudável, esse estudo não realizou ações de educação nutricional, porém vale ressaltar que pesquisas de análises como esta são importantes para diagnóstico do estado nutricional, categorização socioeconômica e educacional, do público, porém não geram impacto a nível de mudança de hábitos alimentares e estilo de vida.

A implementação de políticas de alimentação saudável na escola é uma estratégia de caráter determinante na prevenção e redução da obesidade e outras doenças decorrentes da má alimentação em crianças e adolescentes, porém, o que se observa é que a maioria das escolas 
não executam de maneira adequada estes programas. No estudo Batista et al. (2017) encontraram um alto percentual de crianças com excesso de peso e associado a isso percebeuse uma oferta significativa de alimentos processados e ultraprocessados nos lanches escolares. Assim como Porto et al. (2015) demonstraram em seu estudo que a maioria dos alimentos oferecidos nas cantinas escolares eram industrializados em comparação com somente a presença do suco natural nos cardápios compondo os itens mais vendidos. A escola deve ser um local para oferta de alimentos saudáveis havendo a necessidade de melhorias nos alimentos fornecidos.

A Política de Refeitório Escolar Saudável em Nova Gales do Sul (NSW) exige que as cantinas escolares proíbam a venda de alimentos "vermelhos" ou seja, alimentos que são pobres em nutrientes e ricos em energia, como confeitaria, alimentos fritos e bebidas açucaradas e que a maioria dos itens do cardápio sejam itens "verdes" aqueles com boas fontes de nutrientes, como frutas, legumes, carnes magras (YOONG et al., 2016). Se a escola ofertar alimentos saudáveis possivelmente está contribuindo para redução de sobrepeso e obesidade além da melhoria da saúde dos escolares como mostra o estudo de Ratner et al. (2013) observaram diminuição do percentual de obesidade e as crianças aumentaram o consumo de frutas e legumes, isso pode se dar pela importância de realizar ações com pais, crianças e priorizar venda de alimentos saudáveis no ambiente escolar.

Para aceitabilidade de frutas, legumes e hortaliças no ambiente escolar no estudo Cohen et al. (2015) destacaram algumas estratégias: ofertar primeiro os alimentos mais saudáveis, é uma alternativa que se mostra promissora para adesão desses alimentos, e colocar um chef de cozinha seria uma boa opção para melhorar a palatabilidade dessas preparações de forma a possibilitar a adesão da merenda escolar.

Deve-se introduzir hábitos alimentares corretos precocemente, desde a primeira infância, proporcionando assim, que crianças e adolescentes adquiram bons hábitos alimentares e se tornem adultos mais saudável (LANNES et al., 2018). No estudo de Waters et al. (2018) destacaram que as ações em educação nutricional são mais efetivas quando há o envolvimento não somente dos escolares mas também dos seus pais ou responsáveis, já que estes são encarregados pela alimentação das crianças fora do ambiente escolar, assim como Assis et al. (2014) encontraram no estudo que envolvia alunos, professores, pais e funcionários da cantina, resultados positivos quanto a melhora do conhecimento nutricional e mudanças no comportamento alimentar.

O estudo Cunningham-Sabo; Lohse, (2013) mostra que cozinhar com crianças influência nas suas escolhas alimentares, favorecendo estimular o gosto por frutas e legumes, então incluí a culinária como uma atividade cotidiana da escola com crianças proporciona preferência por alimentos saudáveis através da experimentação direta por meio da degustação 
e do ato de brincar com a comida, já que são atividades que permitem vivenciar o alimento na prática. Assim como Bernardon et al. (2014) demonstraram que as hortas escolares proporcionam experiência de aprendizagem na prática, possibilitando conhecer as características sensoriais e este é um ótimo espaço para desenvolver atividades lúdicas de educação alimentar porém, precisa ser mais explorado pela comunidade escolar pois além de atividades educativas, têm os aspectos da sustentabilidade pois não utiliza agrotóxicos no cultivo, e os alimentos cultivados podem ser usados para complementar a alimentação escolar.

A escola enfrenta algumas dificuldades para alcançar bons resultados de promoção da alimentação saudável, segundo o estudo Waters et al. (2018) citam que as intervenções podem ser limitadas pela influência do marketing vinculado a produtos destinados às crianças que promovem alimentos não saudáveis e está fora do alcance da escola. Assim como Bento et al. (2015) demonstraram em seu estudo que os pais dos escolares utilizavam a televisão como principal meio de obter informações sobre alimentação. Já no estudo Porto et al. (2015) mostram que a pouca interferência da gestão escolar na venda de alimentos nas cantinas escolares favorece aos donos das lanchonetes ofertarem alimentos visando o lucro em detrimento da oferta de alimentos mais saudáveis.

Sendo assim, a escola um espaço social onde crianças e adolescentes passam grande parte do seu tempo, aprendendo e realizando atividades, por tanto é um ambiente propicio para desenvolver bons hábitos alimentares, alcançando estudantes nas etapas mais influenciáveis da sua vida.

\section{Considerações Finais}

Diante disso, vê-se a importância de se realizar ações que promovam e divulguem os alimentos saudáveis, para que as escolas e os gestores tenham o compromisso de executar as políticas de promoção da saúde. Assim, as crianças e os adolescente desde cedo podem se envolver com atividades relacionadas a alimentação e nutrição, visando garantir a prevenção de sobrepeso e obesidade. As metodologias educativas utilizadas como cultivo de hortas escolares, cozinhar com crianças, conhecer o alimento na prática se mostraram mais eficazes do que somente ações expositivas. Constatou-se então que a escola é um espaço ideal para realização destas atividades, principalmente quando existe um elo entre profissionais da saúde e educadores, pois juntos podem definir os melhores métodos de ensino e tornar eficaz a promoção da alimentação saudável, acredita-se que o professor é um grande influenciador de ideias entre os escolares.

Apesar da pesquisa mostrar que Educação Alimentar e Nutricional ganhou espaço nos últimos anos, é preciso ainda formular novas metodologias de ensino, que sejam inovadoras e interativas capazes de facilitar a compreensão dos alunos, desenvolver sua autonomia e 
participação de forma integral, além de aumentar o engajamento de toda a comunidade escolar, pais, professores, gestores e os profissionais da saúde.

\section{Referências}

ARAÚJO, A. L. et al. O impacto da educação alimentar e nutricional na prevenção do excesso de peso em escolares: uma revisão bibliográfica. RBONE-Revista Brasileira de Obesidade, Nutrição e Emagrecimento, v. 11, n. 62, p. 94-105, 2017.

ARAUJO, J. M. et al. Importância Dos Hábitos Alimentares Saudáveis na Infância: uma Revisão da Literatura. International Journal of Nutrology, v. 11, n. S 01, p. Trab458, 2018.

ALVES, G. M. et al. A importância da alimentação saudável para o desenvolvimento humano. Humanas \& Sociais Aplicadas, v. 10, n. 27, p. 46-62, 2020.

BATISTA, M. da S. A. et al. Ações do Programa Saúde na Escola e da alimentação escolar na prevenção do excesso de peso infantil: experiência no município de Itapevi, São Paulo, Brasil, 2014. Epidemiologia e Serviços de Saúde, v. 26, p. 569-578, 2017.

BENTO, I. C. et al. Alimentação saudável e dificuldades para torná-la uma realidade: percepções de pais/responsáveis por pré-escolares de uma creche em Belo Horizonte/MG, Brasil. Ciência \& Saúde Coletiva, v. 20, p. 2389-2400, 2015.

BERNARDON, R. et al. School Gardens in the Distrito Federal, Brazil. Revista de Nutrição, v. 27, n. 2, p. 205-216, 2014.

BRASIL, D. F. Ministério do Desenvolvimento Social e Combate à Fome. Marco de referência de educação alimentar e nutricional para as políticas públicas, MDS; Secretaria Nacional de Segurança Alimentar e Nutricional. 2012.

BRASIL. Decreto presidencial no. 6.286 de 05 de dezembro de 2007. Institui o Programa Saúde na Escola - PSE, e dá outras providências. Brasília (DF): Diário Oficial da União; 2007.

BRASIL. Lei Orgânica de Segurança Alimentar Nutricional (Losan). Lei n 11.346, de 15 de setembro de 2006. Cria o Sistema Nacional de Segurança Alimentar e Nutricional-SISAN com vistas em assegurar o direito humano à alimentação adequada e dá outras providências.

Diário Oficial da União. 2006. Disponível em: https://www.planalto.gov.br/ccivil_03/_Ato2004-2006/2006/Lei/L11346.htm. Acessado em: 16 junho 2018.

BRASIL; BRASIL. Lei n ${ }^{\circ} 11.947$, de 16 de junho de 2009. Dispõe sobre o atendimento da alimentação escolar e do Programa Dinheiro Direto na Escola aos alunos da educação básica. Diário Oficial da União, 2009.

BURITY, V. et al. Direito humano à alimentação adequada no contexto da segurança alimentar e nutricional. Brasília: Abrandh, 2010.

CAMOZZI, A. B. Q. et al. Promoção da Alimentação Saudável na Escola: realidade ou utopia?. Cadernos Saúde Coletiva, v. 23, n. 1, p. 32-37, 2015. 
CHAMBERLAND, K. et al. The impact of an innovative web-based school nutrition intervention to increase fruits and vegetables and milk and alternatives in adolescents: a clustered randomized trial. International Journal of Behavioral Nutrition and Physical Activity, v. 14, n. 1, p. 1-11, 2017.

COHEN, J. F. W et al. Effects of choice architecture and chef-enhanced meals on the selection and consumption of healthier school foods: a randomized clinical trial. JAMA pediatrics, v. 169, n. 5, p. 431-437, 2015.

CUNNINGHAM-SABO, L.; LOHSE, B. Cooking with Kids positively affects fourth graders' vegetable preferences and attitudes and self-efficacy for food and cooking. Childhood Obesity, v. 9, n. 6, p. 549-556, 2013.

DA SILVA, M. X. et al. Abordagem lúdico-didática melhora os parâmetros de educação nutricional em alunos do ensino fundamental. Ciências \& Cognição, v. 18, n. 2, p. 136-148, 2013.

DE ASSIS, M. M. et al. Avaliação do conhecimento nutricional e comportamento alimentar após educação alimentar e nutricional em adolescentes de Juiz de Fora-MG. HU Revista, v. 40, n. 3 e $4,2014$.

FRETES, G. et al. Efecto de una intervención educativa sobre el consumo de frutas, verduras y pescado en familias de niños preescolares y escolares. Archivos latinoamericanos de nutrición, v. 63, n. 1, p. 37-45, 2013.

GREENWOOD, R. L.; FONSECA, A. B. Alimentação na escola: significados e possibilidades do comer e da comida para a promoção dos direitos humanos. Revista Interdisciplinar de Direitos Humanos, v. 6, n. 1, p. 103-127, 2018.

LANNES, M. M. et al. Promoção da saúde no ambiente escolar: desenvolvimento de materiais didáticos. Revista Família, Ciclos de Vida e Saúde no Contexto Social, v. 6, p. 375-385, 2018.

PORTO, E. B. S. et al. School canteens in the Federal District, Brazil and the promotion of healthy eating. Revista de Nutrição, v. 28, n. 1, p. 29-41, 2015.

RATNER, $\mathrm{R}$ et al. Impacto de una intervención en alimentación y nutrición en escolares. Revista chilena de pediatría, v. 84, n. 6, p. 634-640, 2013.

RECH, D. C. et al. As políticas públicas e o enfrentamento da obesidade no Brasil: uma revisão reflexiva. Revista de Epidemiologia e Controle de Infecção, v. 1, n. 1, p. 192-202, 2016.

SANTOS, L. A. da S. O fazer educação alimentar e nutricional: algumas contribuições para reflexão. Ciência \& Saúde Coletiva, v. 17, n. 2, p. 455-462, 2012.

SILVA, M. X. et al. Abordagem lúdico-didática melhora os parâmetros de educação nutricional em alunos do ensino fundamental. Ciências \& Cognição, v. 18, n. 2, p. 136-148, 2013.

SOUSA, L. M. M. S. et al. Metodologia de revisão integrativa da literatura em enfermagem. 2017. 
SOUZA, M. T. de. et al. Revisão integrativa: o que é e como fazer. Einstein (São Paulo), v. 8, n. 1, p. 102-106, 2010.

TRICHES, R. M. Promoção do consumo alimentar sustentável no contexto da alimentação escolar. Trabalho, Educação e Saúde, v. 13, n. 3, p. 757-771, 2015.

WATERS, E. et al. Cluster randomised trial of a school-community child health promotion and obesity prevention intervention: findings from the evaluation of fun ' $n$ healthy in Moreland!. BMC public health, v. 18, n. 1, p. 92, 2018.

YOONG, S. L. et al. CAFÉ: a multicomponent audit and feedback intervention to improve implementation of healthy food policy in primary school canteens: a randomised controlled trial. international journal of behavioral nutrition and physical activity, v. 13, n. 1, p. $126,2016$. 